\title{
Cosmic Bulk Flow and the Local motions from CosmicFlows-2 dataset
}

\author{
H. M. Courtois \\ University of Lyon 1 - CNRS/IN2P3 \\ IPNL 4 rue Enrico Fermi 69622 Villeurbanne, France \\ email: h.courtois@ipnl.in2p3.fr
}

\begin{abstract}
Using the largest and most accurate ever catalog of galaxy peculiar velocities Cosmicflows2 , the large scale structure has been reconstructed by means of the Wiener filter and constrained realizations assuming as a Bayesian prior model the LCDM standard model of cosmology. Our main result is that the estimated bulk flow is consistent with the LCDM model with the WMAP inferred cosmological parameters. At $\mathrm{R}=50(150) \mathrm{Mpc} / \mathrm{h}$ the estimated bulk velocity is $250 \pm$ $21(239 \pm 38) \mathrm{km} / \mathrm{s}$. The SGX and SGY components of the CMB dipole velocity are recovered by the Wiener Filter (WF) velocity field down to a very few $\mathrm{km} / \mathrm{s}$. The SGZ component of the estimated velocity, the one that is most affected by the Zone of Avoidance, is off by $126 \mathrm{~km} / \mathrm{s}$ (an almost 2 sigma discrepancy).
\end{abstract}

\section{Bulk flow}

In the standard model of cosmology, large scale structures grow out of a primordial perturbation field thru gravitational instability. The continuity equation implies that the evolving density field is associated with a peculiar velocity field, both of which represents departures, or fluctuations, from a pure Hubble expansion. The Cosmic Microwave Background dipole anisotropy, which is interpreted as the consequence of the peculiar motion of the Local group with respect to the CMB is the best evidence and example of that motion. Cosmicflows-2 is the second generation catalog of galaxy distances built by the Cosmicflows collaboration. It contains more than 8,000 galaxy peculiar velocities.

The Bulk flow is computed as the mean velocity field in a top-hat sphere as a function of its radius. The amplitude of WF bulk velocity lies within the expected scatter over the range of radius 10 to $150 \mathrm{Mpc}$. This clearly shows the compatibility of the estimated bulk velocity with the CDM/WMAP model.

At $150 \mathrm{Mpc}$ the estimated amplitude is $(239 \pm 38) \mathrm{km} / \mathrm{s}$. It follows that we detect a bulk flow at more than 6 sigma significance at roughly the edge of the data. The flow is mostly within the Zone of Avoidance (ZOA) which almost coincides with the SGX-SGZ plane at $\mathrm{SGY}=0$. The smallest component of the bulk flow is in the SGY direction. The largest component is in the negative SGX direction, detected with a 4.5 sigma significance

We find (Hoffman et al. 2015) that the mean bulk velocity lies within the 1 sigma bracket of the assumed prior model. At $600 \mathrm{Mpc}$ it is $(59 \pm 18) \mathrm{km} / \mathrm{s}$, a 3.2 sigma detection. In the data, there is a non vanishing, at the 1 sigma level, SGX/SGY/SGZ component of the bulk velocity out to radius 400/200/300 Mpc respectively.

\section{Recovering the CMB dipole}

It is well known the direction of bulk velocity remains coherent over a large range of scales. However, is this consistent with the CDM/WMAP model? This question is addressed by calculating the angle between the bulk velocity at a given radius $R$ and its 
direction at $10 \mathrm{Mpc}$. The standard interpretation of the observed dipole moment of the CMB temperature anisotropies is that it is the outcome of our motion with respect to the CMB. The surprising result is how well the flow field recovers the CMB dipole velocity in SGY and in particular in SGX that lies in the Galactic plane. The deficiency of the WF reconstruction in the SGZ direction is expected it is the direction that lies deep in the ZOA and closest to the Galactic center. The misalignment of the Local Group velocity with the observed dipole is found to be $(8 \pm 7$ degrees $)$. However, limiting the study of the alignment in the SGX-SGY plane, namely the Supergalactic Plane, and thereby excluding the SGZ component a much better alignment is found, to be ( $0.4 \pm 5$ degrees).

\subsection{Comparison with similar data and methods}

Nusser \& Davis (2011) analysed the SFI++ data base by means of the All Space Constrained Estimate model. There is a good agreement between the two estimates to within one or two standard deviations of each method. In both cases the estimated bulk velocity is compatible with the cosmic variance predicted by the CDM/WMAP model. Also this agreement in the SGX and SGY directions and the discrepancy in the direction of the Galactic center suggest a minimal role of non-linear dynamics and that the discrepancy between the linear theory estimation and observation is dominated by the obscuration within the ZOA.

\subsection{Malmquist bias is essentially a monopole term}

The Malmquist bias introduces a spurious strong monopole term into the reconstructed velocity field but is expected to hardly affect the bulk velocity which is associated with the dipole of the velocity field. Nevertheless all the analysis presented here was performed on the Malmquist bias corrected data.The comparison shows a very small difference in amplitude at Radius $10 \mathrm{Mpc}$. For Radius $50 \mathrm{Mpc}$ and larger radii, the differences in amplitude amount to a very few $\mathrm{km} / \mathrm{s}$, and the differences in the direction are of the order of a very few degrees. The differences between the outcome of the raw and Malmquist bias corrected data are much smaller than the expected scatter around the WF mean field

\section{Conclusion}

Our current dataset suggests the bulk motion is still present at scale $200 \mathrm{Mpc} / \mathrm{h}$.

The reconstruction has recovered the observed CMB dipole, away from the direction of the Galactic center, suggesting that the CMB has been hardly affected by nonlinear processes. The resulting bulk velocity is consistent with the Bayesian prior CDM/WMAP standard model.

In the next year a third generation of catalog will be ready for extending the analysis a bit further, just reaching out to Shapley supercluster, thanks to deep Green Bank and Arecibo telescopes observations. Also we are adding galaxies observed in NIR with Spitzer space telescope, which allows in particular to reduce the impact of the ZOA. The further step will be to reach a radius of about $300 \mathrm{Mpc}$ using the SKA pathfinders in about 4-5 years. So this is a story to be continued with more beautiful data.

\section{Reference}

Hoffman, Y., Courtois, H. M., \& Tully, R. B. 2015, MNRAS, 449, 4494 